\title{
Robot-assisted arm training in patients with Parkinson's disease: a pilot study
}

\author{
Alessandro Picelli ${ }^{1}$, Stefano Tamburin ${ }^{2}$, Michele Passuello ${ }^{3}$, Andreas Waldner ${ }^{4}$ and Nicola Smania ${ }^{1,5^{*}}$
}

\begin{abstract}
Background: Despite the growing diffusion of robotic devices in neurorehabilitation, no previous study investigated the effects of robotic training on arm impairment due to Parkinson's disease. The aim of this pilot study was to evaluate whether robot-assisted arm training might improve upper limb function in patients with Parkinson's disease.

Findings: Ten patients with Parkinson's disease (Hoehn and Yahr stage 2.5-3) received ten, 45-minute, treatment sessions, five days a week, for two consecutive weeks. Robot-assisted arm training was performed with the Bi-ManuTrack (Reha-Stim, Berlin, Germany) that provides a computer-controlled, repetitive, bilateral, mirror-like practice of forearm pronation/supination and wrist extension/flexion. Patients were trained according to the following modalities: passive-passive (both arms moved by the machine) and active-active (both arms actively moving against resistance). The dominant upper limb was evaluated before and immediately after treatment as well as at two weeks of follow-up. Outcomes were the nine-hole peg test, the Fugl-Meyer assessment (upper limb section) and the Unified Parkinson's Disease Rating Scale. After treatment, a significant improvement was found in the nine-hole peg test $(P=0.007)$ as well as in the upper limb section of the Fugl-Meyer assessment $(P=0.012)$. Findings were confirmed at the 2-week followup evaluation only for the nine-hole peg test $(P=0.007)$. No significant improvement was found in the Unified Parkinson's Disease Rating Scale at both post-treatment and follow-up evaluations.

Conclusions: Our findings support the hypothesis that robot-assisted arm training might be a promising tool in order to improve upper limb function in patients with Parkinson's disease.
\end{abstract}

Keywords: Rehabilitation, Physical therapy, Basal ganglia

\section{Introduction}

Parkinson's disease (PD) is an idiopathic neurodegenerative disorder due to a progressive loss of dopaminergic neurons in the substantia nigra pars compacta [1]. Typical features of PD are bradykinesia (slowed movement), hypokinesia (poverty of movement), rigidity and resting tremor [1,2]. Even if impaired manual dexterity with progressive limitations in reaching, grasping and fine motor tasks has been described in PD, to date evidence base for upper limb intervention strategies in parkinsonian patients is lacking [3].

Robotic arm training (RAT) was found to effectively improve upper limb function in patients with neurological

\footnotetext{
*Correspondence: nicola.smania@univr.it

'Department of Neurological and Movement Sciences, Neuromotor and Cognitive Rehabilitation Research Center, University of Verona, P.le L.A. Scuro, 10, 37134 Verona, Italy

${ }^{5}$ Neurological Rehabilitation Unit, Azienda Ospedaliera-Universitaria Integrata, Verona, Italy

Full list of author information is available at the end of the article
}

disorders, such as stroke [4]. As to PD, forced use, taskspecific, intensive, training programs based on robotic devices were found to effectively improve lower limb function [5-10]. Despite the growing diffusion of robotic devices in neurorehabilitation, to date no previous study investigated the effects of robotic training on arm impairment due to PD. The aim of this pilot study was to evaluate whether RAT might improve upper limb function in patients with PD.

\section{Methods}

This study was performed in the Neurorehabilitation Unit of the Azienda Ospedaliera-Universitaria Integrata of Verona, Italy. Inclusion criteria: confirmed diagnosis of idiopathic PD according to the UK Brain Bank Criteria [11]; Hoehn and Yahr (H\&Y) stage of 2.5 or 3 determined in the "on" phase [12]; Mini Mental State Examination score $>24$ [13]. Exclusion criteria: severe dyskinesias or "on-off" fluctuations; change of PD medication during the

\section{Ciomed Central}


study; deficits of somatic sensation involving the upper limbs; other neurological or orthopedic conditions involving the upper limbs.

All participants were outpatients and gave their informed written consent for participation in the study, which was carried out according to the Declaration of Helsinki and was approved by the Ethics Committee of the Department of Neurological and Movement Sciences of Verona University.

During the study, participants were instructed to take their normal PD medications: they were tested and trained during the on phase ( 1 to 2.5 hours after taking morning dose). Participants did not perform any type of rehabilitation in the three months before the study, nor undergo any form of training other than that scheduled in the study protocol.

\section{Treatment procedures}

Each patient underwent a training program consisting of ten, 45-minute sessions (including rest periods), five days a week (from Monday to Friday) for two consecutive weeks.

Robot-assisted arm training was performed with the Bi-Manu-Track (Reha-Stim, Berlin, Germany) that provides a computer-controlled, repetitive, bilateral, mirrorlike practice of forearm pronation/supination and wrist extension/flexion according to three modalities: passivepassive (both arms moved by the machine), activepassive (one arm driving the other), and active-active (both arms actively moving against resistance) [14]. As shown in Figure 1, patients sat at a height-adjustable table with their elbow bent at $90^{\circ}$, putting their forearms into an arm trough and grasping a handle (written

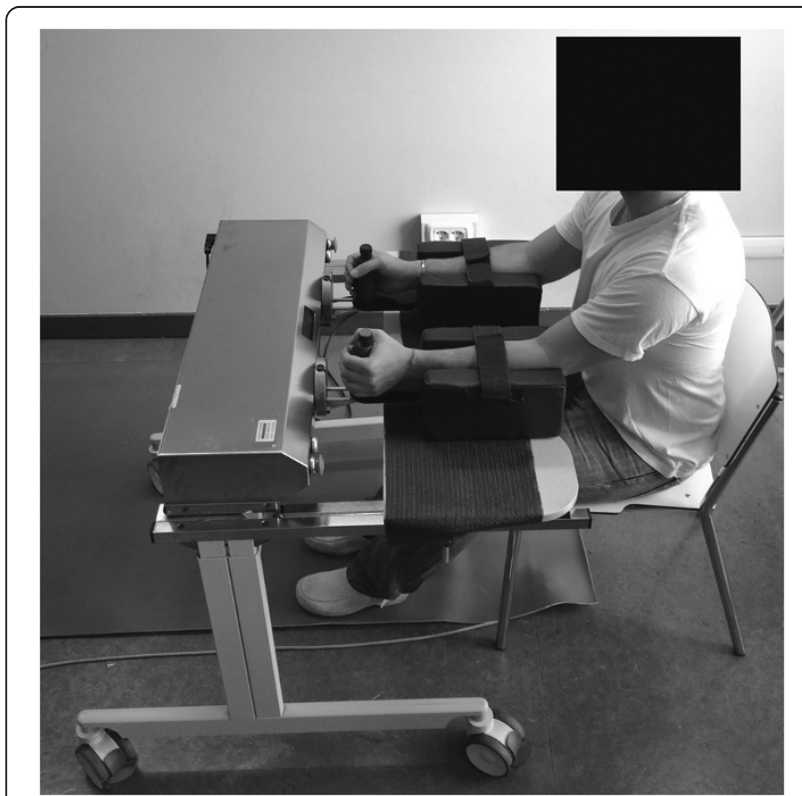

Figure 1 Robot-assisted arm training. informed consent for the publication of this image was obtained). Each training session consisted of two parts with a 5-minute rest between them. First we trained forearm pronation/supination for 20 minutes: $10 \mathrm{mi}-$ nutes of passive-passive mode (100 repetitions) followed by 10 minutes of active-active mode (100 repetitions). Then we trained wrist extension/flexion for 20 minutes: 10 minutes of passive-passive mode (100 repetitions) followed by 10 minutes of active-active mode (100 repetitions). Amplitude and resistance were set individually.

\section{Testing procedures}

Patients were evaluated before (T0), immediately after treatment (T1) (primary endpoint) and at two weeks of follow-up (T2). The same rater evaluated all patients.

\section{Outcome measures}

The nine-hole peg test (NHPT) was used to assess dominant hand dexterity. We required patients to take 9 pegs from a container placing them into 9 holes on a board and vice versa as quickly as possible. Score was the time taken to complete the test activity [15].

The Fugl-Meyer assessment (FM) was used to evaluate dominant upper limb motor ability to perform selective movements. The FM upper limb section allows a maximum score of 66 , with sub-scores of 36 for the upper arm, 10 for the wrist, 14 for the hand, and 6 for coordination and speed of movement $[16,17]$.

The Unified Parkinson's Disease Rating Scale (UPDRS) was used to measure disease severity in PD. It has three subscales: I-mentation, behavior, and mood (range 016); II-activities of daily living (range 0-52); III-motor examination (range $0-108$ ). Total score is the sum of these subscales (range 0-176) $[18,19]$.

\section{Statistical analysis}

Statistical analysis was carried out with SPSS 21.0 (SPSS Inc, Chicago, IL). The Friedman test was used to analyze overall changes in performance between the different evaluation sessions. In the presence of significant main effects, the Wilcoxon signed ranks test was used on the $\mathrm{T} 1$ vs. T0, T2 vs. T0 and T2 vs. T1 comparisons to determine any significant difference. Descriptive analysis was used to evaluate the effect size measures (Cohen's $d$ calculation) and the 95\% confidence intervals [20]. The level for significance was $P<0.05$. The Bonferroni correction was used when investigating multiple comparisons $(P<0.016)[21]$.

\section{Results}

Ten right-handed subjects ( 7 males and 3 females; mean age 70.7 years) presenting with idiopathic PD (mean duration 7.1 years) were recruited from among 18 outpatients consecutively admitted to our Neurorehabilitation Unit 
Table 1 Row data of patients' performance in all outcome measures

\begin{tabular}{|c|c|c|c|}
\hline Outcomes & $\begin{array}{c}\text { Before treatment } \\
\text { Median (IQR) }\end{array}$ & $\begin{array}{l}\text { After treatment } \\
\text { Median (IQR) }\end{array}$ & $\begin{array}{c}\text { Follow-up } \\
\text { Median (IQR) }\end{array}$ \\
\hline Nine-hole peg test $(\mathrm{s})$ & 16.20 (15.80 to 18.18$)$ & 13.80 (13.06 to 14.92$)$ & $13.80(13.08$ to 15.13$)$ \\
\hline Fugl-Meyer assessment (0-66) & $60.00(57.25$ to 62.00$)$ & 65.00 (62.50 to 65.75$)$ & $63.50(62.00$ to 65.75$)$ \\
\hline UPDRS motor examination (0-108) & $16.00(12.25$ to 18.50$)$ & 14.50 (13.25 to 15.75$)$ & $15.00(13.25$ to 16.00$)$ \\
\hline UPDRS total $(0-176)$ & $34.50(25.27$ to 37.75$)$ & 30.00 (24.00 to 34.00$)$ & 27.50 (23.00 to 34.00$)$ \\
\hline
\end{tabular}

Abbreviations: IQR Interquartile range, s seconds, UPDRS Unified Parkinson's Disease Rating Scale.

from April to October 2012. No dropout was observed. No adverse event occurred during the study. Row data (medians and interquartile ranges) of patients' performance at T0, T1 and T2 evaluations are reported in Table 1.

As to the NHPT, a significant overall change was found between the three time points $\left(P=0.007 ; x^{2}=\right.$ 9.800). A significant difference was found at both T1 vs. T0 $(P=0.007)$ and T2 vs. T0 $(P=0.007)$ comparisons. As to the FM, a significant overall change was found between the three time points $\left(P=0.003 ; X^{2}=11.400\right)$. A significant difference was found only at T1 vs. T0 $(P=$ 0.012) comparison. As to the UPDRS, no significant overall change was found between the three time points $\left(P=0.062 ; X^{2}=5.568\right)$ at the Friedman test. Treatment effects in all outcome measures are reported in Table 2.

\section{Discussion}

Our results show that ten, 45-minute sessions of RAT may improve fine and gross motor function of the dominant upper limb in patients with PD (H\&Y 2.5-3). As measured by the NHPT, improvements of fine motor function were maintained also at the follow-up examination. Conversely, no significant change was found in the UPDRS.

In people with $\mathrm{PD}$, altered upper limb function generally manifests as impaired timing and force modulation, progressively affecting the quality of hand movement [3]. Even if intensive, task-specific, practice has been proposed to reduce arm impairment due to $\mathrm{PD}$, the most effective rehabilitative approach in order to best facilitate upper limb skill learning has not yet been defined [3]. Our preliminary findings about the role of RAT in PD are in keeping with those evidences about the effective use of robotic devices provide task-specific, intensive, training programs in patients with progressive lower limb functional impairment due to PD [5-10]. Furthermore, our findings are in line with those of Lee and colleagues, which examined the effects of constraintinduced movement therapy in twenty patients with PD (H\&Y 2-3), observing significant improvements of fine and gross motor performance of the upper limb after twenty, 3-hour, treatment sessions [22].

In order to understand why RAT showed to improve upper limb function in PD, we hypothesize that several repetitions of rhythmic arm movements could act as an external proprioceptive cue, by reinforcing the neuronal circuits that contribute to the upper limb movements. In particular, RAT provides an external rhythm that could improve motor output bypassing the deficient internal motor generation system (including the supplementary motor area and basal ganglia) that would support the generation on actions based on intention and internal reference frame [22]. In addition, it is plausible that the strengthening effect of RAT would play a role. In line with this issue, a previous case series study by Combs and colleagues, described a significantly reduction of disability as well as an improvement of quality of life after a training program based on 24 to 36 boxing sessions in 6 patients with PD (H\&Y 1-4) [23]. Unfortunately, the Authors did not evaluate upper limbs function before

Table 2 Treatment effects in all outcome measures

\begin{tabular}{|c|c|c|c|c|c|c|}
\hline \multirow{4}{*}{ Outcomes } & \multicolumn{3}{|c|}{ Comparisons } & \multicolumn{3}{|c|}{ 95\% Confidence interval } \\
\hline & \multicolumn{3}{|c|}{ Wilcoxon signed ranks test } & \multicolumn{3}{|c|}{ (Effect size) } \\
\hline & T1 vs. T0 & T2 vs. T0 & T2 vs. T1 & T1 vs. T0 & T2 vs. T0 & T2 vs. T1 \\
\hline & $P$ value $(\mathrm{Z})$ & $P$ value $(\mathrm{Z})$ & $P$ value $(\mathrm{Z})$ & $(r)$ & $(r)$ & $(r)$ \\
\hline Nine-hole peg test (s) & $0.007(-2.701)^{*}$ & $0.007(-2.701)^{*}$ & $0.359(-0.918)$ & 1.90 to $4.78(-0.60)$ & 1.73 to $4.34(-0.53)$ & -1.07 to $0.47(0.08)$ \\
\hline Fugl-Meyer assessment $(0-66)$ & $0.012(-2.527)^{*}$ & $0.018(-2.371)$ & $0.606(-0.516)$ & -6.31 to $-1.28(0.45)$ & -5.53 to $-1.26(0.41)$ & -1.62 to $2.42(-0.07)$ \\
\hline $\begin{array}{l}\text { UPDRS motor } \\
\text { examination (0-108) }\end{array}$ & $0.097(-1.658)$ & $0.174(-1.358)$ & $0.334(-0.966)$ & -0.34 to $2.54(-0.14)$ & -0.40 to $2.00(-0.11)$ & -1.05 to $0.45(0.04)$ \\
\hline UPDRS total (0-176) & $0.046(-1.995)$ & $0.037(-2.082)$ & $0.813(-0.214)$ & 0.26 to $6.73(-0.20)$ & 0.54 to $6.65(-0.20)$ & -1.30 to $1.50(-0.01)$ \\
\hline
\end{tabular}


and after treatment [23]. Thus, we cannot directly compare their data with ours.

This pilot study was limited by the lack of a control group and the small sample size. Furthermore there is no long-term follow-up evaluation and no assessment of activities of daily living.

\section{Conclusions}

Our preliminary findings support the hypothesis that RAT might be a promising tool in order to improve upper limb function in patients with PD. However, there is the possibility that changes observed in this pilot study might be due to a placebo effect. Furthermore, it would be useful to evaluate RAT not only in terms of effectiveness but also in terms of costs and time taken to prepare the treatment setting. On these bases, future, properly sized, randomized controlled trials dealing with RAT compared to conventional/non-robotic rehabilitation are needed in order to further validate our results.

\section{Abbreviations}

PD: Parkinson's disease; RAT: Robotic arm training; H\&Y: Hoehn and Yahr; NHPT: Nine-hole peg test; FM: Fugl-Meyer assessment; UPDRS: Unified Parkinson's Disease Rating Scale.

\section{Competing interests}

The authors declare that they have no competing interest.

\section{Authors' contributions}

AP conceived the study, made substantial contribution to its design, performed the statistical analysis and drafted the manuscript. ST participated in the design of the study and helped to draft the manuscript. MP carried out the acquisition of data. AW revised critically the manuscript for important intellectual content. NS coordinated the study and revised critically the manuscript for important intellectual content. All authors read and approved the final manuscript.

\section{Financial disclosure}

The authors received no financial support for the research or authorship of this article.

No commercial party having a direct financial interest in the results of the research supporting this manuscript has or will confer a benefit on the authors or on any organization with which the authors are associated.

\section{Author details}

${ }^{1}$ Department of Neurological and Movement Sciences, Neuromotor and Cognitive Rehabilitation Research Center, University of Verona, P.le L.A. Scuro, 10, 37134 Verona, Italy. ${ }^{2}$ Department of Neurological and Movement Sciences, Neurology Section, University of Verona, Verona, Italy. ${ }^{3}$ Degree Course in Physiotherapy, University of Verona, Verona, Italy. " "Villa Melitta" Rehabilitation Clinic, Bolzano, Italy. ${ }^{5}$ Neurological Rehabilitation Unit, Azienda Ospedaliera-Universitaria Integrata, Verona, Italy.

Received: 4 March 2013 Accepted: 26 February 2014 Published: 5 March 2014

\section{References}

1. Meara J, Koller WC: Parkinson's disease and Parkinsonism in the elderly. Cambridge: Cambridge University Press; 2000.

2. National Institute of Health and Clinical Excellence: Parkinson's disease: diagnosis and management in primary and secondary care. London (UK): Royal College of Physicians; 2006.

3. Quinn L, Busse M, Dal Bello-Haas V: Management of upper extremity dysfunction in people with Parkinson disease and Huntington disease: facilitating outcomes across the disease lifespan. J Hand Ther. in press.
4. Mehrholz J, Hädrich A, Platz T, Kugler J, Pohl M: Electromechanical and robot-assisted arm training for improving generic activities of daily living, arm function, and arm muscle strength after stroke. Cochrane Database Syst Rev 2012, 6, CD006876.

5. Lo AC, Chang VC, Gianfrancesco MA, Friedman JH, Patterson TS, Benedicto DF: Reduction of freezing of gait in Parkinson's disease by repetitive robot-assisted treadmill training: a pilot study. J Neuroeng Rehabil 2010, 7:51.

6. Picelli A, Melotti C, Origano F, Waldner A, Fiaschi A, Santilli V, Smania N: Robot-assisted gait training in patients with Parkinson disease: a randomized controlled trial. Neurorehabil Neural Repair 2012, 26:353-361.

7. Picelli A, Melotti C, Origano F, Waldner A, Gimigliano R, Smania N: Does robotic gait training improve balance in Parkinson's disease? A randomized controlled trial. Parkinsonism Relat Disord 2012, 18:990-993.

8. Carda S, Invernizzi M, Baricich A, Comi C, Croquelois A, Cisari C: Robotic gait training is not superior to conventional treadmill training in parkinson disease: a single-blind randomized controlled trial. Neurorehabil Neural Repair 2012, 26:1027-1034.

9. Picelli A, Melotti C, Origano F, Neri R, Waldner A, Smania N: Robot-assisted gait training versus equal intensity treadmill training in patients with mild to moderate Parkinson's disease: a randomized controlled trial. Parkinsonism Relat Disord 2013, 19:605-610.

10. Smania N, Picelli A, Geroin C, Munari D, Waldner A, Gandolfi M: Robotassisted gait training in patients with Parkinson's disease. Neurodegen Dis Manage 2013, 3:321-330.

11. Hughes AJ, Daniel SE, Kilford L, Lees AJ: Accuracy of clinical diagnosis of idiopathic Parkinson's disease: a clinicopathological study of 100 cases. J Neurol Neurosurg Psychiatry 1992, 55:181-184.

12. Hoehn MM, Yahr MD: Parkinsonism: onset, progression and mortality. Neurology 1967, 17:427-442.

13. Folstein MF, Folstein SE, McHugh PR: Mini-mental state: a practical method for grading the cognitive state of patients for the clinician. J Psychiatr Res 1975, 12:189-198.

14. Hesse S, Schulte-Tigges G, Konrad M, Bardeleben A, Werner C: Robotassisted arm trainer for the passive and active practice of bilateral forearm and wrist movements in hemiparetic subjects. Arch Phys Med Rehabil 2003, 84:915-920.

15. Oxford Grice K, Vogel KA, Le V, Mitchell A, Muniz S, Vollmer MA: Adult norms for a commercially available nine hole peg test for finger dexterity. Am J Occup Ther 2003, 57:570-573.

16. Fugl-Meyer AR, Jääskö L, Leyman I, Olsson S, Steglind S: The post-stroke hemiplegic patient. 1. a method for evaluation of physical performance. Scand J Rehabil Med 1975, 7:13-31.

17. Lee KS, Lee WH, Hwang S: Modified constraint-induced movement therapy improves fine and gross motor performance of the upper limb in Parkinson disease. Am J Phys Med Rehabil 2011, 90:380-386.

18. Fahn S, Elton RL: UPDRS program members. Unified Parkinsons Disease Rating Scale. In Recent developments in Parkinsons disease, Volume 2. Edited by Fahn S, Marsden CD, Goldstein M, Calne DB. Macmillan Healthcare Information: Florham Park, NJ; 1987:153-163.

19. Shulman LM, Gruber-Baldini AL, Anderson KE, Fishman PS, Reich SG, Weiner WJ: The clinically important difference on the unified Parkinson's disease rating scale. Arch Neurol 2010, 67:64-70.

20. Cohen J: Statistical power analysis for the behavioral sciences. 2nd edition. Lawrence Erlbaum: Hillsdale, NJ; 1988.

21. Bryant TN, Machin D: Statistical methods. In Rehabilitation studies handbook. Edited by Wilson BA, McLellan DL. Cambridge, UK: Cambridge University Press; 1997:189-204.

22. Nieuwboer A, Rochester L, Müncks L, Swinnen SP: Motor learning in Parkinson's disease: limitations and potential for rehabilitation. Parkinsonism Relat Disord 2009, 15(Suppl 3):53-58.

23. Combs SA, Diehl MD, Staples WH, Conn L, Davis K, Lewis N, Schaneman K: Boxing training for patients with Parkinson disease: a case series. Phys Ther 2011, 91:132-142.

\section{doi:10.1186/1743-0003-11-28}

Cite this article as: Picelli et al:: Robot-assisted arm training in patients with Parkinson's disease: a pilot study. Journal of NeuroEngineering and Rehabilitation 2014 11:28. 